\title{
Material Assessment for ITER's Collective Thomson Scattering first mirror
}

Santos, R. ; Policarpo, H. ; Gonçalves, B. ; Varela, P. ; Nonbøl, Erik; Klinkby, Esben Bryndt; Lauritzen, Bent; Romanets, Y. ; Luis, R. ; Vaz, P.

Published in:

Proceedings of Advancements in Nuclear Instrumentation Measurement Methods and their Applications 2015

Publication date:

2015

Document Version

Peer reviewed version

Link back to DTU Orbit

Citation (APA):

Santos, R., Policarpo, H., Gonçalves, B., Varela, P., Nonbøl, E., Klinkby, E. B., Lauritzen, B., Romanets, Y., Luis, R., \& Vaz, P. (2015). Material Assessment for ITER's Collective Thomson Scattering first mirror. In Proceedings of Advancements in Nuclear Instrumentation Measurement Methods and their Applications 2015

\section{General rights}

Copyright and moral rights for the publications made accessible in the public portal are retained by the authors and/or other copyright owners and it is a condition of accessing publications that users recognise and abide by the legal requirements associated with these rights.

- Users may download and print one copy of any publication from the public portal for the purpose of private study or research.

- You may not further distribute the material or use it for any profit-making activity or commercial gain

- You may freely distribute the URL identifying the publication in the public portal 


\section{Material Assessment for ITER's Collective Thomson Scattering first mirror}

CONFERENCE PAPER · APRIL 2015

DOWNLOADS

10

10 AUTHORS, INCLUDING:

Hugo Policarpo

Technical University of Lisbon

26 PUBLICATIONS 71 CITATIONS

SEE PROFILE

\section{R. Luís}

Technical University of Lisbon

6 PUBLICATIONS 10 CITATIONS

SEE PROFILE
VIEWS

20

Bent Lauritzen

Technical University of Denmark

44 PUBLICATIONS 748 CITATIONS

SEE PROFILE

Pedro Vaz

Instituto Superior Técnico, University of Lisb...

441 PUBLICATIONS 1,639 CITATIONS

SEE PROFILE 


\title{
Material Assessment for ITER’s Collective Thomson Scattering first mirror
}

\author{
R. Santos ${ }^{1}$, H. Policarpo ${ }^{1}$, B. Gonçalves ${ }^{1}$, P. Varela ${ }^{1}$, E. Nonbøl ${ }^{2}$, E. Klinkby ${ }^{2}$, B. Lauritzen ${ }^{2}$, Y. Romanets ${ }^{3}$, R. Luis ${ }^{3}$, \\ P. Vaz
}

Abstract- ITER's Collective Thomson Scattering (CTS) system is a diagnostic instrument that will measure the plasma density and velocity through Thomson scattering of microwave radiation. Some of the key components of the CTS are quasioptical mirrors used to produce astigmatic beam patterns, which have impact on the strength and spatial resolution of the diagnostic signal. The mirrors are exposed to neutron radiation, which may alter the mirror properties or deform its structure. These changes may affect the collection of the scattered radiation and consequently decrease the quality of the measurements.

In this work, three different materials (molybdenum (Mo), stainless steel 316L (SS-316L) and tungsten (W)) are considered for the first mirror of the CTS. The objective is to assess the suitability of these materials for this mirror and to provide a first ranking, considering the neutron radiation loads requirements defined by ITER, based on the resultant maximum Von Misses stresses and temperatures. For it, the neutron irradiation, and subsequent heat-load on the mirrors were simulated using the Monte Carlo N-Particle (MCNP) code. Based on the MCNP heat-load results, a transient thermal-structural Finite Element Analysis (FEA) of the mirror over a 400s discharge (reasonable number for computational tests, since an ITER discharge will be between $200 \mathrm{~s}$ and $1000 \mathrm{~s}$ ), with and without mirror cooling, is performed.

The results obtained in this preliminary analysis show that of the tested materials $M o$ and $W$ are the most suitable materials for this application, being able to reliably sustain the thermal and structural stresses imposed by the neutron loads.

\section{INTRODUCTION}

COLLECTIVE Thomson Scattering is a microwave plasma diagnostic, which aims at measuring the phase space

Manuscript received April 6, 2015. This work was supported in part by the Framework Partnership agreement (F4E-FPA393) between IPFN and/ DTURisØ supported by F4E.

${ }^{1}$ Authors are associated with Instituto de Plasmas e Fusão Nuclear, Instituto Superior Técnico, Universidade de Lisboa, 1049-001 Lisboa, Portugal (corresponding author - telephone: +351 218419086, e-mail: rsantos@ipfn.ist.utl.pt).

${ }^{2}$ Authors are associated with Center for Nuclear Technologies, Technical University of Denmark.

${ }^{3}$ Authors are associated with Centro de Ciências e Tecnologias Nucleares, Instituto Superior Técnico, Universidade de Lisboa, Portugal. densities of confined fast ions populations, as well as the 1-D fast ion velocity distribution [1]. These fast ion populations have energies in the $\mathrm{MeV}$ range and carry approximately 1/3 of the plasma kinetic energy. So, it is necessary to guarantee that there is not a significant loss of these fast ions. These losses may occur due to instabilities in the plasma and may cause damage to the tokamak walls, as well as the plasma disruption [2].

The CTS system at ITER will be developed by the Denmark Technical University (DTU) in a partnership with Instituto de Plasmas e Fusão Nuclear (IPFN). The system will be located on the Low Field Side (LFS) of ITER. The millimeter wave generator will be a $1 \mathrm{MW}$ radiofrequency power gyrotron. It launches the radiation into the plasma, which is then reflected and redirected, through a set of mirrors, to the horn antennas [3].

The CTS first mirror, with preliminary dimensions of $1020 \mathrm{~mm}$ in length, $300 \mathrm{~mm}$ in height and $100 \mathrm{~mm}$ thick, is a fundamental component of the entire system. Its function is to redirect the reflected radiation from the plasma to other mirrors. The main concern with the first mirror is related with assuring its integrity under the neutron fluxes to which it is exposed, as it may affect the quality of the acquired signal [4]. Based on ITER's material list [12], candidate materials are: Mo, SS-316L and $\mathrm{W}$ are here considered for the first mirror of the CTS to access which of these materials are best suited, considering the different neutron radiation loads required by ITER. For it, neutronics analysis are performed, using the MCNP code for particle simulations, to estimate the energy deposited through the volume of the mirror and the flux of particles that cross the surface of the mirror. These quantities are considered as thermal loads in the transient FEA of the mirror (performed using commercial software ANSYS) over a 400s discharge, with and without cooling on the rear side for which, a Finite Element (FE) model of the mirror is developed using 3D thermal-structural FEs.

Results show that the maximum stress and temperature of the mirror, considering Mo and W, present satisfactory values as they are below the respective yield stress and melting point temperature.

SS-316L may be considered in future studies, in which lower cooling temperatures and/or additional cooling areas of the mirror are considered.

This study may serve as an initial, but essential, mirror material suitability analysis that may be considered in following and more intricate studies. If we compare the work on this paper to [1], there is here a new dynamic FEA analysis, the ranking of different materials suggested by ITER and a change from the LFS to the HFS. 


\section{Collective ThOMSON SCATtering PHYSics}

The CTS system on ITER will be located on port plug \#12 and its preliminary design has already been defined by ITER, as illustrated in Fig. 1.

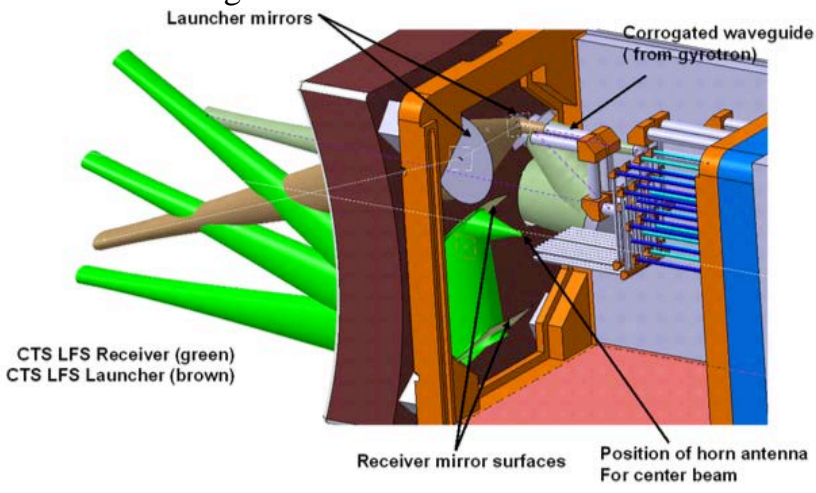

Fig. 1 - Ris $\varnothing$ 's engineering design proposal for the CTS system [4].

Fig. 1 comprises the fundamental elements of a CTS system, i.e., the launcher and receiver components. Each of these has their respective beams, mirrors, antennas and waveguides.

The interaction between the millimeter wave and the plasma is based on the Bragg condition

$$
\boldsymbol{k}^{s}=\boldsymbol{k}^{i}+\boldsymbol{k}^{\delta},
$$

where $\boldsymbol{k}^{s}$ is the scattered wave vector, $\boldsymbol{k}^{i}$ the incident wave vector and $\boldsymbol{k}^{\delta}$ the resolved direction wave vector. The incident wave is scattered on the density wave and origins the scattered wave, which is measured by the experimental setup. The measured quantities are resolved along the density wave direction.

The transfer equation of a generic CTS system [5] may be expressed as

$$
\frac{\partial P^{S}}{\partial \nu^{s}}=P^{i} O_{b}\left(\lambda_{0}^{i}\right)^{2} r_{e}^{2} n_{e} \frac{1}{2 \pi} \Sigma,
$$

where $P^{i}$ is the power of the incident wave, $O_{b}$ the beam overlap zone in the plasma, $\lambda_{0}^{i}$ the incident wave wavelength, $r_{e}$ the electron radius, $n_{e}$ the electron density and $\Sigma$ the scattering function.

\section{NEUTRONICS ANALYSIS}

The radiation impact over the first mirror of the CTS is considered as a flux and its average value over a surface or inside a volume. The heat flux may be expressed as

$$
F_{2}=\frac{1}{A} \int_{A} d A \int_{E} d E \int_{d \Omega} 4 \pi \phi\left(r_{s}, E, \Omega\right),
$$

where $A$ is the surface area of the mirror, $E$ the energy of each particle passing through the mirror and $\Omega$ the cross section.

Furthermore, the heat generation inside the mirror is also considered and may be expressed

$$
F_{6}=\frac{\rho_{a}}{V \rho_{g}} \int_{V} \int_{t} \int_{E} H(E) \phi(r, E, t) d E d t d V,
$$

where $t$ represents the discharge time, $V$ the volume, $\rho_{a}$ the atomic density, $\rho_{g}$ the mass density, $\phi(r, E, t)$ the particle flux and $H(E)$ the heating response.
These quantities are estimated using MCNP and are used as inputs in the FEA.

\section{FINITE ELEMENT ANALYSIS}

Transient thermal-structural FEA of the mirror are conducted over a 400s discharge, for which the heat and Navier's continuum equations are solved. A homogeneous and isotropic 3D model of the mirror is developed using a 20 node 3D thermal-structural FEs with four degrees-of-freedom (displacements and temperature) at each node (see, SOLID226 [8]).

The thermal loads considered in the FEA are the heat flux, expressed by Eq.(3), heat generation, expressed by Eq.(4), and a radiation heat flux, from the mirror to the surrounding assuming gray body radiation with an emissivity of 0.5.

Furthermore, when cooling is considered a constant temperature load of $473 \mathrm{~K}$ is applied to the back side of the mirror.

A structural load with zero displacement is additionally considered in the back of the mirror to simulate its physical support.

Partial integral differential equations are discretized on a regular mesh composed of 20000 brick elements as illustrated in Fig. 2.

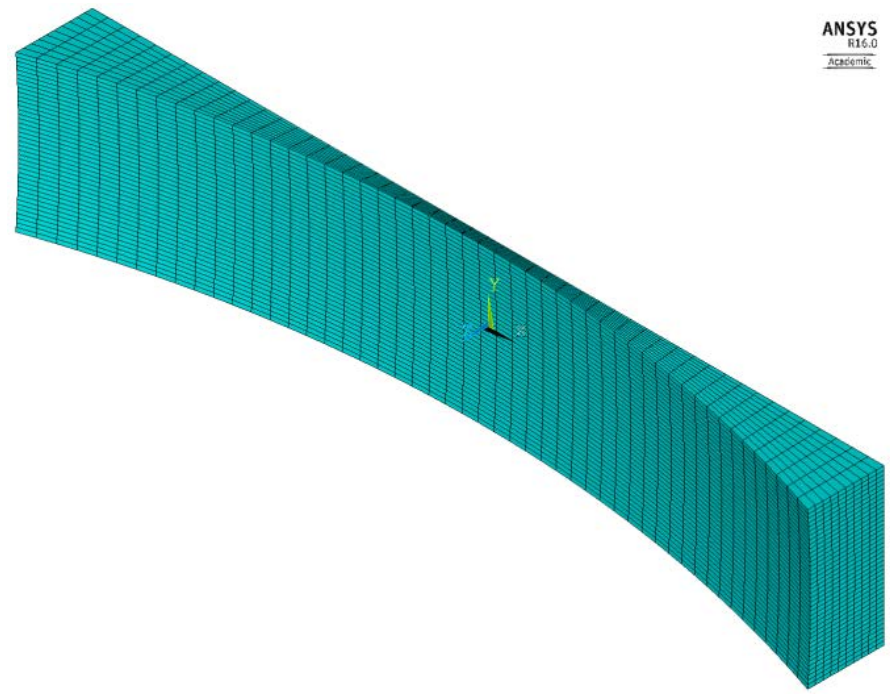

Fig. 2 - FE mesh of the first mirror of the CTS.

The thermal loads are applied at the initial time step and the set of equations are solved using the preconditioned conjugate gradient solver in which Newmark's algorithm [9] is used for time integration.

\section{RESULTS AND DISCUSSION}

The materials considered for the first mirror of the CTS system are Mo, SS-316L and $\mathrm{W}$ with properties presented in Table 1. 
TABLE I. MATERIAL PROPERTIES USED IN THE FEA

\begin{tabular}{cccc} 
Materials & Mo & SS-316L & W \\
\hline Mass Density (g/cm $\left.{ }^{3}\right)$ & 10.22 & 7.95 & 19.30 \\
Specific Heat (J/Kg.K) & 255 & 560 & 134 \\
Thermal Conductivity & 138 & 16 & 163 \\
(W/m.K) & & & \\
Young’s Modulus (GPa) & 330 & 184 & 400 \\
Melting Point (K) & 2896 & 1647 & 3273 \\
Yield Stress (MPa) & 400 & 170 & 550 \\
\hline
\end{tabular}

The neutronics analysis is performed using the MCNP code, as described in section III.

The radiation impact over the mirror, i.e., the heat flux through the surface of the mirror, determined by Eq.(3), and the heat generated in the volume of the mirror due to the particle irradiation, determined by Eq.(4), are presented in Table II for the different materials.

TABLE II. HEAT LOADS AND HEAT FLUXES OF THE MATERIALS.

\begin{tabular}{cccc} 
Materials & Mo & SS-316L & W \\
\hline Heat Flux $\left(\mathrm{W} / \mathrm{m}^{2}\right)$ & 1.21 & 1.25 & 1.01 \\
Heat load $\left(\mathrm{MW} / \mathrm{m}^{3}\right)$ & 8.00 & 3.47 & 0.98 \\
\hline
\end{tabular}

Taking these values as inputs for the FEA of the mirror, as described in section IV, one obtains the maximum Von Mises stress and temperature over the ITER's time discharge of 400s with and without cooling.

Fig. 3 illustrates the maximum temperatures obtained along the discharge time with cooling (dashed lines) and without cooling (solid lines). The results obtained considering Mo are presented by the cross marked line, SS-316L are presented by the triangle marked line and $\mathrm{W}$ are presented by the square marked line.

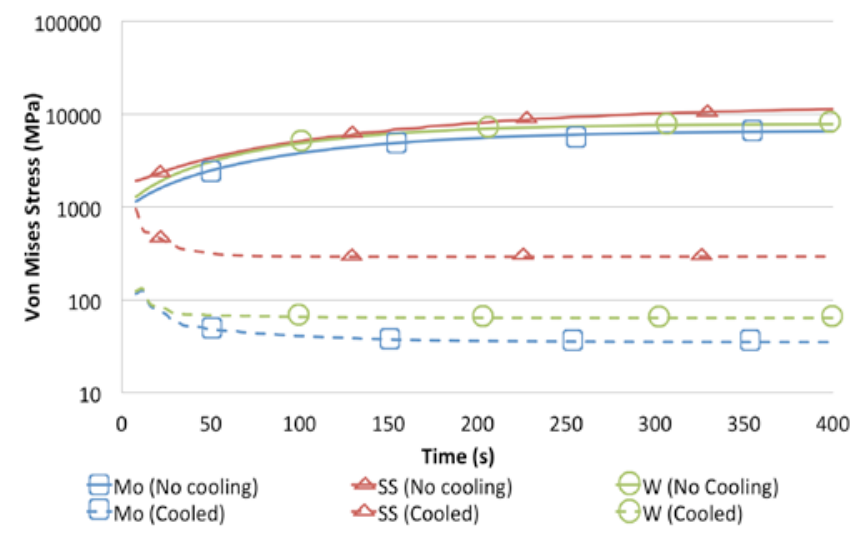

Fig. 3 - Maximum temperature obtained along the discharge time for the different materials with and without cooling.

In Fig. 3 it is possible to observe that without cooling (solid lines) the temperatures are higher, as expected, relatively to the case with cooling (dashed lines). After the discharge time of 400s the mirror composed of Mo, SS-316L and W, for the case without cooling, reaches a temperature of $1572 \mathrm{~K}, 1575 \mathrm{~K}$ and $1783 \mathrm{~K}$, respectively.
For each of these materials, at the maximum temperatures obtained, there is the possibility of microstructures changes that may affect the resultant mechanical properties, which may not be acceptable.

Hence, cooling on the back side of the mirror, as described in section IV, is considered.

In this case, and for the same discharge time of 400s the mirror composed of Mo, SS-316L and W reach temperatures of $487 \mathrm{~K}, 1114 \mathrm{~K}$ and $545 \mathrm{~K}$, respectively.

Furthermore, it is observed that the temperature of the SS316L mirror increases over the duration of a discharge, whereas for Mo and W mirrors is constant, at the level of the cooling temperature of the mirror. This is due to the fact that Mo and $\mathrm{W}$ have higher thermal conductivities (approximately 8 and 10 times higher, respectively) to the SS-316L (see Table II).

Hence, Mo followed by W and SS-316L would be the rank of the most suitable materials, among those here considered, for the mirror if a minimum temperature criterion is considered.

However, to complete the picture, Vons Mises stresses should also be considered.

Fig. 4 illustrates the maximum Von Misses stresses obtained along the discharge time with cooling (dashed lines) and without cooling (solid lines). The results obtained using Mo are represented by the cross marked line, SS-316L are represented by the triangle marked line and $\mathrm{W}$ are represented by the square marked line.

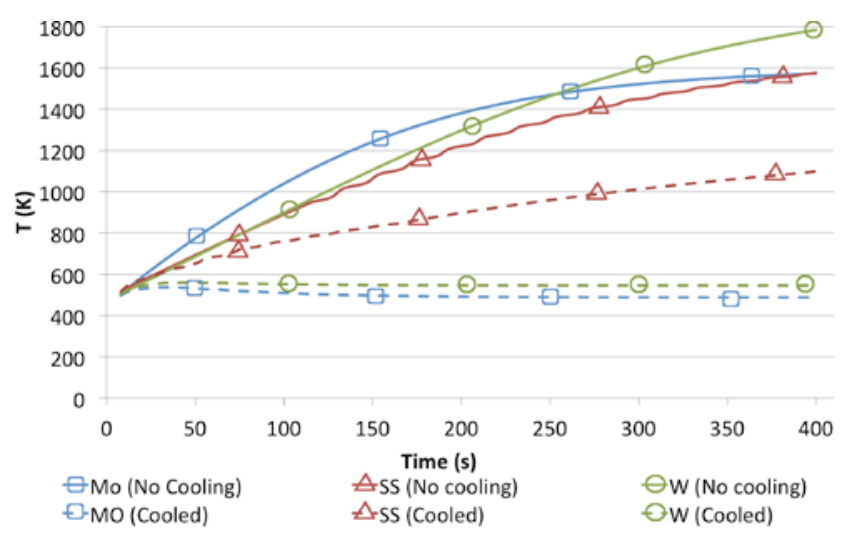

Fig. 4 - Maximum Von Misses stresses obtained along the discharge time for the different materials with and without cooling.

Fig. 4 shows that without cooling (solid lines) the maximum Von Misses stresses increase rate and are higher, as expected, relative to the case with cooling (dashed lines). After the discharge time of 400s the mirror composed of Mo, SS-316L and $\mathrm{W}$, for the case without cooling, present maximum Von Misses stresses of $6.54 \mathrm{GPa}, 11.40 \mathrm{GPa}$ and $7.83 \mathrm{GPa}$, respectively.

Since these values surpass the yield stress of all the materials considered, see Table I, one may conclude that the mirror cannot sustain the load requirements of ITER without cooling.

When cooling is considered, and for the same discharge time of 400s the mirror composed of Mo, SS-316L and W, 
present maximum Von Misses stresses of 35MPa, 294MPa and 64MPa, respectively. Thus, Mo and $\mathrm{W}$ mirrors present maximum Von Misses stresses below the yield stresses of the respective materials, as presented in Table I.

On the other hand, even when cooling is considered, SS316L presents a maximum Von Misses stress above the yield stress, as presented in Table I, and for this reason, it may be discarded as a viable material.

Hence, of the tested materials, Mo followed by W may be considered as the most suitable materials for the first mirror of the CTS. Mirrors made of either one of these materials present maximum Von Misses stresses bellow the correspondent yield stresses and maximum temperatures below the respective melting point temperatures and those that would allow for phase transitions.

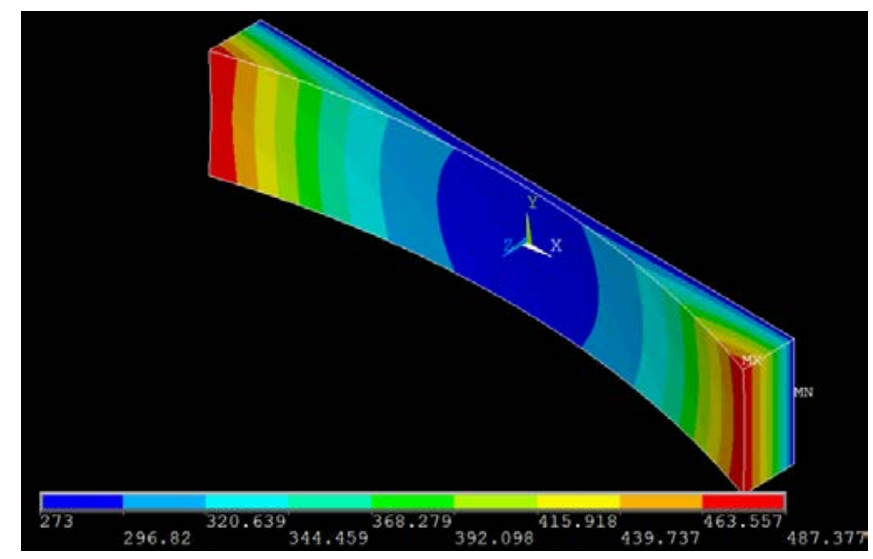

Fig. 5 - FEA temperature distribution for the Mo first mirror of the CTS.

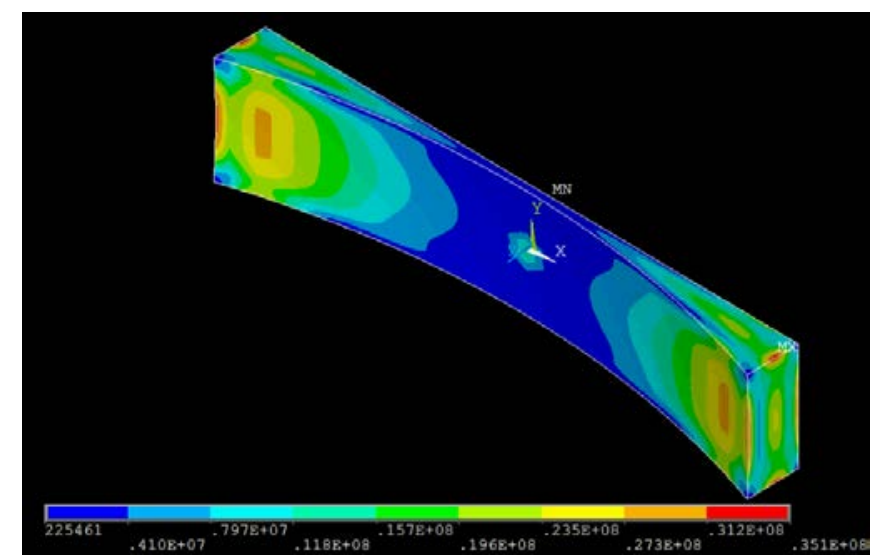

Fig. 6 - FEA Von Mises stress distribution for the Mo first mirror of the CTS.

In this sense, Fig. 5 and Fig. 6 illustrate the temperature and Von Misses stress distribution of the Mo first mirror of the CTS. Note that the stress and temperature distributions over the mirror are similar for the other materials, varying only its magnitude.

The maximum temperature and Von Misses stress of $1114 \mathrm{~K}$ and 294MPa, respectively, occur on the top and bottom lateral sides of the mirror, as illustrated by Fig. 5 and Fig. 6.

\section{CONCLUSIONS}

Recalling that the main concern with the first mirror of the CTS is related with assuring its integrity under the neutron fluxes to which it is exposed, results show that of the tested materials Mo followed by $\mathrm{W}$ are the most suitable materials for this application due to their mechanical properties that allow for maximum temperatures and stresses values below the maximum admissible.

Even though SS-316L does not respect the maximum allowable temperatures and stresses, future studies considering the use of SS-316L in which lower cooling temperatures and/or additional cooling areas are considered are suggested.

This paper presents an initial and essential analysis on the CTS's system first mirror materials. It follows a previously used approach to the problem [1], adding an analysis over the discharge time of an ITER shot. So, these improvements represent a step forward to the goal of the first mirror material definition.

The future work will have to include FEA analysis considering that the material properties vary with temperature.

This was not an essential task on this work, since we wanted to rank materials instead of making a rigorous and time-consuming analysis.

Furthermore, the DTU team had access to information that opens a possibility for a new material to be considered. This

material is Zirconium ( $\mathrm{Zr}$ ) and is not present on the default ITER materials [10] for first mirrors.

\section{ACKNOWLEDGMENTS}

This work was supported by F4E in the context of the Framework Partnership Agreement between IPFN and DTURisØ. Fundação para a Ciência e Tecnologia (FCT) also was responsible for financial support of working members of the project.

The views and opinions expressed herein do not necessarily reflect those of the European Commission.

\section{REFERENCES}

[1] M. Salewski, F. Meo, H. Bindslev, V. Furtula, S. B. Korsholm et. al.,

[2] Investigation of the First Mirror Heating for the Collective Thomson Scattering Diagnostic in ITER, Rev. Sci. Instrum., 2008.

[3] H. Bindslev, Collective Thomson Scattering for Diagnosis of Fast Ions, Special Lecture at the $8^{\text {th }}$ International Symposium on Laser-Aided Plasma Diagnostics, Proceedings, September 1997, Page 265.

[4] P. K. Michelsen et. al., Engineering Design of the ITER Collective

[5] Thomson Scattering Diagnostic, Risø Report, 1717, pages 5-9, 2009.

[6] M. Rubel et. al., An overview of a comprehensive First Mirror Test for ITER at JET, Journal of Nuclear Materials 390-391 (2009) 1066-1069.

[7] H. Bindslev et. al., Microwave diagnostics, Fusion Science and Technology Vol. 53 (2009) 1066-1069.

[8] H. Bindslev et. al., ITER Fast Ion Collective Thomson Scattering, Association EUROATOM-Risø, 2003.

[9] X-5 Monte Carlo Team, MCNP - A General Monte Carlo N-Particle Transport Code, Los Alamos National Laboratory, 2003.

[10] Ansys Help Manual (version 16).

[11] N. M. Newmark, A Method of Computation for Structural Dynamics, J. Eng. Mech. Div. ASCE, Vol. 85, No. 3 (1959) 67-94.

[12] J.W. Davis and P.D. Smith, ITER material properties handbook, Journal of Nuclear Materials, Vol. 233-237, Part 2 (1996) 1593-1596. 